\title{
Accélérer notre intervention : Plan d'action quinquennal du gouvernement du Canada sur les infections transmissibles sexuellement et par le
}

\section{sang}

\author{
C Jackson'1, G Tremblay ${ }^{1}$ au nom du Comité directeur du Plan d'action du gouvernement du \\ Canada sur les ITSS*
}

\section{Résumé}

Les infections transmissibles sexuellement et par le sang (ITSS), dont le VIH, I'hépatite B et $\mathrm{C}$, la chlamydia, la gonorrhée, la syphilis et le virus du papillome humain, demeurent un problème important de santé publique tant à l'échelle nationale qu'internationale. En 2018, les gouvernements fédéral, provinciaux et territoriaux ont publié un Cadre d'action pancanadien sur les ITSS (le Cadre) visant à définir une approche globale et exhaustive de lutte contre les ITSS à l'intention de toutes les personnes intervenant sur cette problématique. Ce groupe comprend des ordres de gouvernement, les communautés et dirigeants des Premières Nations, des Inuits et des Métis, les fournisseurs de services de première ligne, les cliniciens, les spécialistes de la santé publique, les organisations non gouvernementales et les chercheurs. Le Cadre fixe des objectifs stratégiques, des principes directeurs et des axes d'action pour lutter contre les ITSS au Canada. En réponse, le gouvernement du Canada a publié son propre plan d'action en juillet 2019 : Accélérer notre intervention : Plan d'action quinquennal du gouvernement du Canada sur les infections transmissibles sexuellement et par le sang (le Plan d'action). Ce document distingue sept domaines sur lesquels le gouvernement fédéral focalisera son action au cours des cinq prochaines années pour lutter contre les ITSS:

1) Progresser vers la vérité et la réconciliation avec les Premières Nations, les Inuits et les Métis; 2) Stigmatisation et discrimination; 3) Innovation communautaire - mettre l'accent sur la prévention; 4) Rejoindre les personnes non diagnostiquées - accroître l'accès au dépistage des ITSS; 5) Offrir des services de prévention, de traitement et de soins aux populations qui reçoivent des services de santé ou des prestations de soins de santé du gouvernement fédéral; 6) Mettre à profit les connaissances actuelles et orienter la recherche future; 7) Mesurer l'impact - assurer le suivi et rendre compte des tendances et des résultats. Présentement, le gouvernement du Canada collabore avec les gouvernements provinciaux et territoriaux, les partenaires des Premières Nations, des Inuits et des Métis et les autres intervenants à l'élaboration, dans le contexte canadien, d'indicateurs et d'objectifs en lien avec les ITSS qui soient adaptés, réalisables et mesurables par rapport aux objectifs stratégiques communs du Cadre et du Plan d'action. Par ailleurs, le gouvernement du Canada s'est engagé à rendre compte chaque année des progrès accomplis dans la mise en œuvre des domaines d'action prioritaires énoncés dans le Plan d'action.
Cette oeuvre est mise à la disposition selon les termes de la licence internationale Creative Commons Attribution 4.0

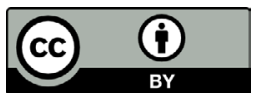

Affiliation

${ }^{1}$ Centre de la lutte contre les maladies transmissibles et les infections, Agence de la santé publique du Canada, Ottawa, ON

${ }^{\star}$ Correspondance:

ccdic-clmti@phac-aspc.gc.ca

Citation proposée : Jackson C, Tremblay G au nom du Comité directeur du Plan d'action du gouvernement du Canada sur les ITSS. Accélérer notre intervention : Plan d'action quinquennal du gouvernement du Canada sur les infections transmissibles sexuellement et par le sang. Relevé des maladies transmissibles au Canada 2019;45(12):355-9. https://doi.org/10.14745/ccdr.v45i12a04f

Mots-clés : ITSS, plan d'action, cadre, Canada

\section{Introduction}

Les infections transmissibles sexuellement et par le sang (ITSS), dont le VIH, I'hépatite B et C, la chlamydia, la gonorrhée, la syphilis et le virus du papillome humain, demeurent une importante préoccupation de santé publique au Canada. Au Canada, les taux d'infections transmissibles sexuellement ont augmenté de manière spectaculaire entre 2008 et 2017 : les taux 
de chlamydia, de gonorrhée et de syphilis ont respectivement augmenté, de $39 \%, 109 \%$ et $167 \%$ (1). Ces dernières années, plusieurs territoires ont signalé des éclosions de syphilis; le nombre de cas de syphilis congénitale s'est également accru (2-4). Bien que le nombre de décès imputables au $\mathrm{VIH}$ et les cas d'hépatite $\mathrm{C}$ signalés ont fortement diminué depuis les années 1990, de nouvelles infections continuent à apparaître $(5,6)$. Globalement, il y a une dynamique en faveur de l'élimination des ITSS en tant que préoccupation de santé publique, et le Canada a adopté les objectifs mondiaux de I'Organisation mondiale de la santé en ce qui concerne le VIH, I'hépatite virale et les infections transmissibles sexuellement. Ces infections ont des voies de transmission et des facteurs de risque en commun et, bien souvent, les populations les plus touchées ou les plus exposées se chevauchent.

Améliorer la prévention, le dépistage, le traitement et la prise en charge des ITSS est une tâche complexe qui fait intervenir un certain nombre d'acteurs, dont l'ensemble des ordres de gouvernement, les communautés et dirigeants des Premières Nations, des Inuits et des Métis (encadré), les fournisseurs de services de première ligne, les cliniciens, les spécialistes de la santé publique, les organisations non gouvernementales et les chercheurs. En 2018, l'Agence de la santé publique du Canada a publié un Cadre d'action pancanadien sur les ITSS (le Cadre) (7) qui a été adopté par les ministères fédéral, provinciaux et territoriaux de la Santé. Ce Cadre définit une approche globale et exhaustive de lutte contre les ITSS à l'intention de toutes les personnes intervenant sur cette problématique. II précise les objectifs stratégiques, les principes directeurs et les axes d'action tout au long du continuum de soins relatifs aux ITSS, et met en évidence le rôle des environnements (conditions sociales, culturelles, physiques, structurelles et juridiques) propices à la prévention de la transmission des ITSS et à l'amélioration de I'accès aux services. Le Cadre a été résumé dans un précédent article du Relevé des maladies transmissibles au Canada (8).

\section{Encadré : Ministères fédéraux associés au Plan d'action quinquennal du gouvernement du Canada sur les infections transmissibles sexuellement et par le sang: \\ - Agence de la santé publique du Canada \\ - Instituts de recherche en santé du Canada \\ - Service correctionnel du Canada \\ - Ministère de la Justice \\ - Ministère de la Défense nationale \\ - Ministère des Femmes et de l'Égalité des genres \\ - Santé Canada \\ - Immigration, Réfugiés et Citoyenneté Canada \\ - Services aux Autochtones Canada \\ - Bureau du Conseil privé - Secrétariat LGBTQ2}

En juillet 2019, le gouvernement du Canada (GC) a publié son propre plan décrivant ses priorités phares pour réduire les répercussions des ITSS sur la santé publique au Canada et faire progresser les travaux engagés en lien avec le Cadre entre 2019 et 2024. Ce plan, intitulé Accélérer notre intervention : Plan d'action quinquennal du gouvernement du Canada sur les infections transmissibles sexuellement et par le sang (le Plan d'action) (9) adopte une approche pangouvernementale et fait intervenir dix ministères fédéraux qui, par leur mandat et leurs activités, ciblent divers aspects des ITSS ou sont chargés de fournir des services de prévention, de traitement ou de soins, ou des prestations de soins de santé à certaines populations.

Ce plan partage les objectifs stratégiques du Cadre. Ces objectifs consistent à (1) réduire l'incidence des ITSS au Canada, (2) améliorer l'accès au dépistage, au traitement, aux soins de santé et au soutien et (3) réduire la stigmatisation et la discrimination qui accentuent la vulnérabilité face aux ITSS. L'article suivant résume le Plan d'action et décrit brièvement ses sept domaines d'action prioritaires.

\section{Renouveler l'intervention fédérale face aux ITSS : vue d'ensemble du Plan d'action}

\author{
Le Plan d'action contient sept domaines d'action prioritaires.
}

\section{Progresser vers la vérité et la réconciliation avec les Premières Nations, les Inuits et les Métis}

Cet engagement du Plan d'action reflète l'engagement plus large du GC en faveur de la réconciliation. Le GC adoptera une approche concernant les politiques, les programmes et les services en matière d'ITSS nécessaires pour répondre aux priorités des communautés des Premières Nations, des Inuits et des Métis. Dans le cadre de ce domaine d'action prioritaire, le GC appuiera les priorités des Premières Nations, des Inuits et des Métis en lien avec les ITSS, améliorera la disponibilité et I'accessibilité des données communautaires sur les résultats en matière d'ITSS, et investira dans des initiatives de prévention, d'éducation et de sensibilisation adaptées aux réalités culturelles pour faciliter l'accès aux soins et au soutien continus.

\section{Stigmatisation et discrimination}

La stigmatisation et la discrimination restent des obstacles majeurs à une prévention, une prise en charge, un traitement et un soutien efficaces en matière d'ITSS. Les populations confrontées à différentes formes de stigmatisation et de discrimination, dont le sexisme, le racisme et l'homophobie, sont touchées de manière disproportionnée par les ITSS. La stigmatisation et la discrimination empêchent également ces personnes d'accéder à des services adaptés de prévention, de soin, de traitement et de soutien concernant les ITSS. Le GC promouvra le message important «indétectable $=$ intransmissible» $(I=\mid)$. Celui-ci repose sur un ensemble substantiel de données scientifiques indiquant que les personnes vivant avec le $\mathrm{VIH}$ ayant atteint une charge virale indétectable de manière durable ne présentent aucun risque de transmission 
sexuelle. Dans le cadre de ce domaine d'action prioritaire, le GC s'emploiera également à :

- lutter contre la stigmatisation et la discrimination (notamment le racisme et le sexisme), la violence fondée sur le sexe, la transphobie, la biphobie et l'homophobie

- doter les professionnels des compétences nécessaires pour fournir des services adaptés aux réalités culturelles dans des environnements sécuritaires

- réduire la criminalisation de la non-divulgation de la séropositivité au $\mathrm{VIH}$ au Canada

\section{Innovation communautaire - mettre l'accent sur la prévention}

Le GC poursuivra son soutien de longue date aux organismes communautaires qui œuvrent à prévenir les ITSS au moyen de programmes et d'interventions adaptés aux contextes et aux priorités des communautés concernées. Ce soutien se traduit notamment par les programmes fédéraux de subventions et de contributions, dont le Fonds de réduction des méfaits (10) et le Fonds d'initiatives communautaires en matière de VIH et d'hépatite $C(11)$. Dans le cadre de ce domaine d'action prioritaire, le GC soutiendra les projets communautaires de première ligne pour prévenir les nouvelles infections et les infections récurrentes, atteindra les personnes non diagnostiquées et les orientera vers des services de dépistage, de traitement et de soins, et accompagnera les efforts visant à transposer à grande échelle les interventions à forte incidence, comme la prophylaxie préexposition pour le VIH, pour qu'un plus grand nombre de personnes puissent en bénéficier.

\section{Rejoindre les personnes non diagnostiquées - accroître l'accès au dépistage des ITSS}

Le dépistage des ITSS permet aux personnes qui s'y soumettent d'accéder à des soins, à un traitement et à un soutien et peut, à terme, réduire le taux de transmission des infections. Cependant, les approches actuelles en matière de dépistage des ITSS ne permettent pas d'atteindre toutes les personnes susceptibles d'en bénéficier. C'est pourquoi, dans le cadre de ce domaine d'action prioritaire, le GC accordera la priorité aux efforts visant à accroître l'accès aux modèles de dépistage qui sont culturellement sécurisants et à élargir les options de dépistage proposées au Canada, notamment le dépistage hors laboratoire, le dépistage autonome et le dépistage par analyse de gouttes de sang séché.

5. Offrir des services de prévention, de traitement et de soins aux populations qui reçoivent des services de santé ou des prestations de soins de santé du gouvernement fédéral

Le GC finance et fournit des services de santé ou des prestations de soins de santé à certaines populations, notamment les membres des Forces armées canadiennes, à certaines populations d'immigrants, aux personnes incarcérées dans les établissements correctionnels fédéraux ainsi qu'aux membres des Premières Nations et aux Inuits admissibles. Dans le cadre de ce domaine d'action prioritaire, le GC veillera à ce que les personnes admissibles reçoivent des services efficaces et culturellement appropriés en ce qui concerne la prévention (y compris la réduction des méfaits), le dépistage et le traitement, et facilitera l'acheminement vers les soins pour les personnes qui passent du système de santé fédéral aux systèmes de santé des provinces ou des territoires.

\section{Mettre à profit les connaissances actuelles et orienter la recherche future}

La recherche scientifique continue d'améliorer les connaissances sur les ITSS, les paramètres favorisant la vulnérabilité face aux infections, l'élaboration de nouvelles modalités de dépistage, de prévention et de traitement, ainsi que la connaissance des répercussions physiques, mentales et sociales sur les personnes atteintes d'une infection chronique. Dans le cadre de ce domaine d'action prioritaire, le GC investira dans : la recherche fondamentale, translationnelle et clinique; la recherche en appui à la prévention, au dépistage, au diagnostic, au traitement et à la prise en charge des ITSS; le renforcement des capacités de recherche en santé des Premières Nations, des Inuits et des Métis.

\section{Mesurer l'impact - assurer le suivi et rendre compte des tendances et des résultats}

Les objectifs mondiaux concernant le $\mathrm{VIH}$, I'hépatite et les infections transmissibles sexuellement s'inscrivent dans le cadre de la volonté du Canada de réduire les répercussions des ITSS sur la santé. Néanmoins, les objectifs mondiaux ne suffisent pas pour fédérer les efforts, susciter le changement et mesurer les progrès accomplis par le Canada. Dans le cadre de ce domaine d'action prioritaire, le GC pilotera un processus visant à élaborer des indicateurs et des objectifs adaptés au contexte canadien en ce qui concerne les ITSS. II donnera également la priorité aux efforts destinés à renforcer le système canadien de surveillance des ITSS pour s'assurer qu'il dispose des données nécessaires pour focaliser son action et suivre les tendances et les changements au fil du temps. Le GC s'est également engagé à rendre compte chaque année des résultats obtenus avec ce Plan d'action.

\section{Conclusion et prochaines étapes}

Le Plan d'action décrit les sept domaines prioritaires sur lesquels le gouvernement focalisera son action pour mettre en œuvre le Cadre et aider le Canada à satisfaire aux objectifs mondiaux concernant le VIH, I'hépatite et les infections transmissibles sexuellement d'ici 2030 (tableau 1). Le GC collabore actuellement avec les gouvernements provinciaux et territoriaux, les partenaires des Premières Nations, des Inuits et des Métis et les autres intervenants à l'élaboration, dans le contexte canadien, d'indicateurs et d'objectifs adaptés, réalisables et mesurables en ce qui concerne les ITSS. Ce travail nécessitera la tenue d'une consultation communautaire dans les mois à venir. Le GC s'est également engagé à rendre compte chaque année des progrès accomplis dans la mise en œuvre des domaines d'action prioritaires énoncés dans le Plan d'action. La pleine mise 
en œuvre du Cadre exigera néanmoins des efforts continus et concertés de la part des gouvernements, des intervenants et des communautés concernées pour veiller à ce que des services de prévention, de dépistage, de soins, de traitement et de soutien adaptés aux réalités culturelles soient accessibles aux personnes qui en ont besoin.

\section{Tableau 1 : Objectifs mondiaux en matière d'infections transmissibles sexuellement et par le sang (ITSS)}

\begin{tabular}{|c|c|c|}
\hline ITSS & $\begin{array}{c}\text { Année } \\
\text { cible }\end{array}$ & Objectifs mondiaux \\
\hline \multirow[t]{2}{*}{$\mathrm{VIH}$} & 2020 & $\begin{array}{l}\text { - } 90 \% \text { des personnes vivant avec le VIH } \\
\text { connaissent leur état sérologique } \\
\text { - } 90 \% \text { des personnes vivant avec le VIH } \\
\text { qui connaissent leur état sérologique } \\
\text { suivent un traitement } \\
\text { - } 90 \% \text { des personnes suivant un } \\
\text { traitement ont une charge virale } \\
\text { indétectable } \\
\text { - moins de } 500000 \text { nouvelles infections } \\
\text { au VIH } \\
\text { - élimination de la discrimination liée au } \\
\text { VIH }\end{array}$ \\
\hline & 2030 & $\begin{array}{l}\text { - Aucune nouvelle infection au VIH } \\
\text { - Aucun décès lié au sida } \\
\text { - Aucune discrimination }\end{array}$ \\
\hline \multirow[t]{2}{*}{ Hépatite } & 2020 & $\begin{array}{l}\text { - } 30 \% \text { moins de nouveaux cas d'hépatites } \\
\text { virales chroniques B et } C \\
\text { - } 10 \% \text { moins de décès imputables aux } \\
\text { hépatites B et } C \\
\text { - } 30 \% \text { des hépatites virales B et } C \text { sont } \\
\text { diagnostiquées } \\
\text { - cinq millions de personnes suivent } \\
\text { un traitement contre l'hépatite } B \text { et } \\
\text { trois millions de personnes suivent un } \\
\text { traitement contre l'hépatite } C \\
\text { - atteinte et maintien d'une couverture } \\
\text { vaccinale de } 90 \% \text { pour le vaccin contre } \\
\text { l'hépatite B (trois doses) }\end{array}$ \\
\hline & 2030 & $\begin{array}{l}\text { - } 90 \% \text { moins de nouveaux cas d'hépatites } \\
\text { virales chroniques B et } C \\
\text { - } 65 \% \text { moins de décès imputables aux } \\
\text { hépatites B et } C \\
\text { - } 90 \% \text { des hépatites virales B et } C \text { sont } \\
\text { diagnostiquées } \\
\text { - } 80 \% \text { des personnes admissibles } \\
\text { reçoivent un traitement contre les } \\
\text { hépatites B et } C\end{array}$ \\
\hline $\begin{array}{l}\text { Infections } \\
\text { transmissibles } \\
\text { sexuellement }\end{array}$ & 2030 & $\begin{array}{l}\text { - diminution de } 90 \% \text { de l'incidence de la } \\
\text { - dyphilis à l'échelle mondiale } \\
\text { diminution de } 90 \% \text { de l'incidence de la } \\
\text { gonorrhée à l'échelle mondiale } \\
\text { - } 50 \text { cas ou moins de syphilis congénitale } \\
\text { pour } 100000 \text { naissances vivantes dans } \\
80 \% \text { des pays } \\
\text { - maintien d'une couverture nationale } \\
\text { de } 90 \% \text { et d'au moins } 80 \% \text { dans } \\
\text { chaque district (ou unité administrative } \\
\text { équivalente) dans les pays où le vaccin } \\
\text { contre le virus du papillome humain } \\
\text { fait partie du programme national } \\
\text { d'immunisation }\end{array}$ \\
\hline
\end{tabular}

La réussite de ces efforts repose sur les partenariats. Le GC s'engage à mettre en œuvre les Appels à l'action de la
Commission de vérité et réconciliation (12) et continuera à travailler en étroite collaboration avec les communautés et les dirigeants des Premières Nations, des Inuits et des Métis. Le GC s'engage également à continuer à travailler avec les personnes qui ont une expérience vécue au cours du développement des programmes et les politiques. Enfin, le travail que le GC accomplit en collaboration avec les provinces, les territoires et les organismes communautaires reste essentiel à l'atteinte des objectifs stratégiques communs en matière d'ITSS. Avec son Plan d'action, le gouvernement fédéral continuera à accomplir des progrès dans la lutte contre le $\mathrm{VIH}$, I'hépatite virale et les infections transmissibles sexuellement dans le contexte du Cadre.

\section{Déclaration des auteurs}

C. J. - Rédaction et édition

G. T. - Rédaction et édition

\section{Conflit d'intérêts}

Aucun.

\section{Remerciements}

L'Agence de la santé publique du Canada tient à remercier toutes les personnes qui ont mis leur temps et leur expertise au service de l'élaboration du Plan d'action.

\section{Références}

1. Agence de la santé publique du Canada. Rapport sur les infections transmissibles sexuellement au Canada, 2017. Ottawa (ON) : ASPC; 2019. https://www.canada.ca/fr/ sante-publique/services/publications/maladies-et-affections/ rapport-infections-transmissibles-sexuellement-canada-2017. html

2. Choudhri Y, Miller J, Sandhu J, Leon A, Aho J. La syphilis infectieuse et la syphilis congénitale au Canada, de 2010 à 2015. Relevé des maladies transmissibles au Canada 2018 Feb;44(2):47-53. DOI

3. The Society of Obstetricians and Gynaecologists of Canada. SOGC Infectious Diseases Committee Statement on Syphilis. Ottawa (ON): SOGC; 2019. https://www.sogc.org/ en/content/featured-news/sogc-infectious-diseases-com mittee-statement-on-syphilis.aspx

4. Agence de la santé publique du Canada. Syphilis infectieuse au Canada, 2018. Relevé des maladies transmissibles au Canada 2019;45(11):332. https://www. canada.ca/content/dam/phac-aspc/documents/services/ reports-publications/canada-communicable-disease-reportccdr/monthly-issue/2019-45/issue-11-november-7-2019/ ccdrv45i11a05f-fra.pdf 
5. Haddad N, Li JS, Totten S, McGuire M. Le VIH au Canada, Rapport de surveillance, 2017. Relevé des maladies transmissibles au Canada 2018 Dec;44(12):367-76. DOI

6. Agence de la santé publique du Canada. Nombre de cas signalés de maladies de 1924 à 2017 au Canada- maladies à déclaration obligatoire en direct. Hépatite $C$ : Taux par 100000 des cas signalés de maladies au fil du temps au Canada, groupé par maladie. Ottawa (ON) : ASPC; 2019. (Accédé 2019-10-03). https://maladies.canada.ca/ declaration-obligatoire/graphiques?c=pl

7. Agence de la santé publique du Canada. Réduction des répercussions sur la santé des infections transmissibles sexuellement et par le sang au Canada d'ici 2030 : un cadre d'action pancanadien sur les ITSS. Ottawa (ON) : ASPC; 2018 juin. https://www.canada.ca/ $\mathrm{fr} /$ sante-publique/services/maladies-infectieuses/ sante-sexuelle-infections-transmissibles-sexuellement/ rapports-publications/infections-transmissible s-sexuellement-sang-cadre-action.html

8. Centre de la lutte contre les maladies transmissibles et les infections. Résumé du cadre pancanadien sur les infections transmissibles sexuellement et par le sang. Relevé des maladies transmissibles au Canada 2018;44(7/8):201-4. DOI
9. Agence de la santé publique du Canada. Accélérer notre intervention : plan d'action quinquennal du gouvernement du Canada sur les infections transmissibles sexuellement et par le sang. Ottawa (ON) : ASPC; 2019 juillet. https://www. canada.ca/fr/sante-publique/services/rapports-publications/ accelerer-notre-intervention-plan-action-quinquennal-infecti ons-transmissibles-sexuellement-sang.html

10. Agence de la santé publique du Canada. Fonds pour la réduction des méfaits; Prévenir la transmission du VIH et de l'hépatite $C$ dû au partage de matériel d'injection et d'inhalation de drogues. Ottawa, ON : ASPC; 2019. https://www.canada.ca/fr/sante-publique/services/ occasions-financement/fonds-reduction-mefaits.html

11. Agence de la santé publique du Canada. Fonds d'initiatives communautaires en matière de $\mathrm{VIH}$ et d'hépatite $\mathrm{C}$. Ottawa, ON : ASPC; 2019. https://www.canada.ca/fr/sante-publique/ services/occasions-financement/fonds-initiative s-communautaires-matiere-vih-sida-hepatite-prochainesetapes.html

12. Commission de vérité et du réconciliation du Canada Centre national pour la vérité et la réconciliation : Appels à l'action. 2015. https://nctr.ca/fr/assets/reports/Final\%20Reports/ Calls_to_Action_French.pdf 